\title{
Successful application of focused extracorporeal shockwave therapy for plantar fasciitis in patients suffering from metastatic breast cancer
}

\author{
Andrej Zdravkovic ${ }^{1} \cdot$ Michael Mickel $^{1} \cdot$ Richard Crevenna $^{1}$ (it \\ Received: 14 December 2020 / Accepted: 26 February 2021 / Published online: 21 March 2021 \\ (C) The Author(s) 2021
}

\begin{abstract}
Purpose Focused extracorporeal shock wave therapy (fESWT) has been shown to be effective in a large number of musculoskeletal disorders. Until 2016, cancer was considered a contraindication for fESWT. The goal of this Commentary is to address the subject of fESWT in cancer patients and present a case of a successful application of fESWT in a breast cancer patient with metastatic bone disease, suffering from debilitating heel pain caused by plantar fasciitis.

Methods The subject of fESWT application in cancer patients is discussed using the example of a 75-year-old female with breast cancer and metastatic bone disease suffering from bilateral inferior heel pain, who was referred to our clinic with a tentative diagnosis of polyneuropathy. Patient history, clinical examination, electrodiagnostic testing, and radiological findings all indicated plantar fasciitis, rather than polyneuropathy. The possibility of metastatic bone lesions in the treatment area was excluded and the patient was thereupon treated with 5 weekly applications of low-energy fESWT.

Results The treatment lead to a reduction in pain of approximately $80 \%$ with no adverse events.

Conclusion fESWT may be a viable treatment option for plantar fasciitis even in cancer patients, provided certain conditions are met.
\end{abstract}

Keywords Extracorporeal shockwave therapy $\cdot$ Plantar fasciitis $\cdot$ Breast neoplasms $\cdot$ Metastatic bone disease

\section{Introduction}

Extracorporeal shock wave therapy (ESWT) is a physical therapy modality in which pressure waves are generated and transmitted to body tissues [1]. Of the two existing types, namely focused and radial ESWT, only focused ESWT (fESWT) produces pressure waves, which have the typical characteristics of shockwaves [2]. The proposed mechanisms of action of ESWT include pain relief, possibly by means of hyperstimulation analgesia and the stimulation of tissue regeneration, in part due to increased matrix turnover and collagen production [1].

ESWT is successfully used to treat plantar fasciitis (PF) [3]. Although adverse effects are rare [4], ESWT is often neglected as a treatment option in cancer patients, as cancer

Richard Crevenna

richard.crevenna@meduniwien.ac.at

1 Department of Physical Medicine, Rehabilitation and Occupational Medicine, Medical University of Vienna, Waehringer Guertel 18-20, A-1090 Vienna, Austria was widely considered a contraindication for ESWT. In 2016, the International Society for Medical Shockwave Treatment issued a consensus statement on ESWT indications and contraindications, where tumors in the treatment area were classified as a contraindication, but not cancer per se as an underlying disease [5].

In this case study, the successful treatment of PF in a patient suffering from breast cancer with metastatic bone disease is described.

\section{Case presentation}

\section{Patient history}

A 75-year-old Caucasian female was referred to the Department of Physical Medicine, Rehabilitation and Occupational Medicine of the Medical University of Vienna with a diagnosis of polyneuropathy. The patient described a bilateral heel pain with a gradual onset, beginning approximately 4 months before the referral. The pain was of an undulating intensity, with maxima reaching $81 \mathrm{~mm}$ on a visual 
analog scale while walking upon getting out of bed. As the painful area was located medially on the heel, the patient was forced to put her weight on the lateral rim of the foot while walking.

The walking ability was severely reduced, with a maximum uninterrupted walking distance of approximately 100 $\mathrm{m}$. The limiting factor was a pain in the lateral compartment of the left knee, which had become apparent about the same time as the heel pain. However, recurring knee pain had been known for several years before the referral.

She was suffering from breast cancer, which was diagnosed in 2014 and had been treated with surgery, followed by adjuvant chemotherapy. Metastatic bone disease was ascertained at the time of diagnosis and consisted of lesions in the spine, pelvic bones, and several ribs. No recent progression of the underlying disease had been observed.

\section{Clinical presentation}

In the clinical examination, the heel pain was localized on both sides along the medial part of the insertion of the plantar aponeurosis on the calcaneus, as well as along the most medial bundle of its central part. Pressure on these areas elicited pain of the same quality and location as experienced during walking.

A recent bone scintigraphy showed no tracer uptake in the feet. Uptake in the medial condyle of the left femur and of the left tibia was consistent with osteoarthritis.

Plain radiographs revealed a plantar heel spur on both sides, without indication of bone lesions. The plain radiograph of the left calcaneus is shown in Fig. 1.

Ultrasound showed a thickening of the plantar aponeurosis near the insertion on the calcaneus, with a thickness of $4.9 \mathrm{~mm}$ on the left side and $4.8 \mathrm{~mm}$ on the right side.

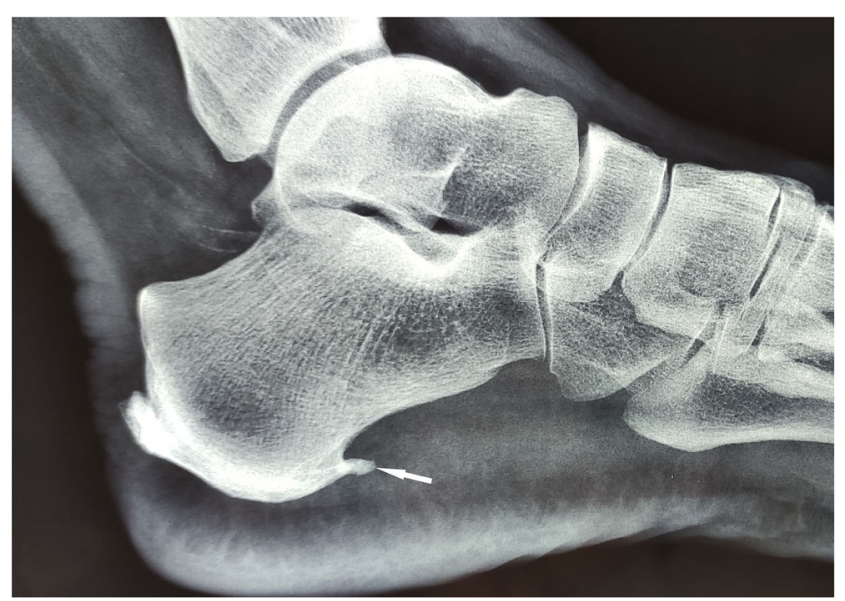

Fig. 1 Plain radiograph of the left calcaneus. Arrow indicates plantar heel spur
Polyneuropathy was excluded through electrodiagnostic testing.

\section{Intervention}

fESWT was administered on both heels over 5 weekly sessions using the F10G4 therapy source of a PiezoWave 2 device (Richard Wolf GmbH, Knittlingen, Germany). One thousand five hundred impulses were administered on each foot with a frequency of $5 \mathrm{~Hz}$, at a focus depth of $15 \mathrm{~mm}$ and an average energy flow density of approximately $0.39 \mathrm{~mJ} / \mathrm{mm}^{2}$. The focus depth was chosen according to the thickness of the tissues superficial to the aponeurosis, measured during ultrasound imaging.

fESWT did not cause any adverse events. The reported pain intensity decreased gradually after each session. At week 6 , the patient reported a maximum pain intensity of $14 \mathrm{~mm}$ on the visual analog scale. The patient reported no pain in the heels up to 12 weeks after fESWT, during which time she received physical therapy for knee pain due to osteoarthritis.

\section{Discussion}

The patient was referred to our clinic with a tentative diagnosis of polyneuropathy and a substantial disease burden caused by bilateral heel pain. The initial diagnosis of polyneuropathy was not in line with the results of electrodiagnostic testing, and clinical and radiological findings. Although polyneuropathy cannot be considered a "zebra" in a cancer patient, there are usually multiple "horses" to be considered. Indeed, PF is the most common cause of inferior heel pain [6]. However, metastatic disease of the foot, though exceedingly rare, should be excluded in patients with malignant disease and foot pain [7].

In older adults, PF has been associated with a greatly increased prevalence of falls [8]. In addition, cancer patients seem to be at an increased risk of falling, and prior falls are associated with a worse outcome of cancer therapy $[9,10]$.

ESWT is an effective treatment option for PF [3] and the presence of a heel spur in lateral radiographs may present a positive prognostic factor concerning the success of ESWT [11]. Historically, cancer per se was considered a contraindication for ESWT, and this position changed only recently, following a consensus statement issued by the German Speaking International Society for Extracorporeal Shockwave Treatment [12], as well as the International Society for Medical Shockwave Treatment [5]. As exercise represents an important adjunct therapy with a substantial beneficial impact in cancer management [13], it stands to reason that an effective treatment of painful musculoskeletal 
disorders is of great importance in relation to the implementation of exercise guidelines.

Functional limitations, which in turn reduce the healthrelated quality of life, are highly prevalent in adults living with cancer, and a reduced walking ability may affect more than half of this patient group [14]. These limitations should therefore be treated with a specific, targeted treatment plan, in order to restore or at least improve the physical, and thusly psychosocial functioning of patients. At our hospital, an interdisciplinary, multimodal approach is the standard in terms of cancer rehabilitation and supportive care [15].

Osteoporosis, a condition for which breast cancer survivors are at an increased risk [16], is not considered a contraindication for fESWT [5]. Indeed, initial findings suggest a positive effect of fESWT on bone formation [17].

In our experience, cancer patients can profit from a multimodal approach to side-effect management and supportive care, and ESWT plays an important role in the treatment of a number of disorders, including PF [18-20].

\section{Conclusion}

fESWT seems to be a safe, efficient, and cost-effective treatment option in cancer patients with PF and can be administered in an outpatient setting, provided that no metastatic lesions are present in the treatment area.

Code availability Not applicable.

Funding Open access funding provided by Medical University of Vienna.

Data Availability All background information concerning the methodology of the intervention and creation of this paper is open for journal review if requested.

\section{Declarations}

Ethics approval The described therapeutic intervention was conducted within the scope of routine clinical work at the Department of Physical Medicine, Rehabilitation and Occupational Medicine of the Medical University of Vienna.

Consent to participate The patient provided verbal informed consent to the participation.

Consent for publication The patient provided verbal informed consent to the consequent publication of this article, provided the data were anonymized.

Conflict of interest The authors declare no competing interests.
Open Access This article is licensed under a Creative Commons Attribution 4.0 International License, which permits use, sharing, adaptation, distribution and reproduction in any medium or format, as long as you give appropriate credit to the original author(s) and the source, provide a link to the Creative Commons licence, and indicate if changes were made. The images or other third party material in this article are included in the article's Creative Commons licence, unless indicated otherwise in a credit line to the material. If material is not included in the article's Creative Commons licence and your intended use is not permitted by statutory regulation or exceeds the permitted use, you will need to obtain permission directly from the copyright holder. To view a copy of this licence, visit http://creativecommons.org/licenses/by/4.0/.

\section{References}

1. van der Worp H, van den Akker-Scheek I, van Schie H, Zwerver J (2013) ESWT for tendinopathy: technology and clinical implications. Knee Surg Sport Traumatol Arthrosc 21:1451-1458

2. Cleveland RO, Chitnis PV, McClure SR (2007) Acoustic field of a ballistic shock wave therapy device. Ultrasound Med Biol 33: $1327-1335$

3. Aqil A, Siddiqui MRS, Solan M, Redfern DJ, Gulati V, Cobb JP (2013) Extracorporeal shock wave therapy is effective in treating chronic plantar fasciitis: a meta-analysis of RCTs. Clin Orthop Relat Res 471:3645-3652

4. Roerdink RL, Dietvorst M, Zwaard BVD, van der Worp H, Zwerver J (2017) Complications of extracorporeal shockwave therapy in plantar fasciitis: systematic review. Int J Surg 46:133-145

5. Eid J (2016) Consensus statement on ESWT indications and contraindications. [cited 2020 Apr 9]. Available from: https://www. shockwavetherapy.org/fileadmin/user_upload/dokumente/PDFs/ Formulare/ISMST_consensus_statement_on_indications_and contraindications_20161012_final.pdf

6. Van Leeuwen KDB, Rogers J, Winzenberg T, Van Middelkoop M (2016) Higher body mass index is associated with plantar fasciopathy/'plantar fasciitis': systematic review and metaanalysis of various clinical and imaging risk factors. Br J Sports Med 50:972-981

7. Maheshwari AV, Chiappetta G, Kugler CD, Pitcher JD Jr, Temple HT (2008) Metastatic skeletal disease of the foot: case reports and literature review. Foot Ankle Int 29:699-710

8. Menz HB, Auhl M, Spink MJ (2018) Foot problems as a risk factor for falls in community-dwelling older people: a systematic review and meta-analysis. Maturitas. 118:7-14

9. Wildes TM, Dua P, Fowler SA, Miller JP, Carpenter CR, Avidan MS, Stark S (2015) Systematic review of falls in older adults with cancer. J Geriatr Oncol 6:70-83

10. Maltser S, Cristian A, Silver JK, Morris GS, Stout NL (2017) A focused review of safety considerations in cancer rehabilitation. PM R 9:S415-S428

11. Yin M, Chen N, Huang Q, Marla AS, Ma J, Ye J, Mo W (2017) New and accurate predictive model for the efficacy of extracorporeal shock wave therapy in managing patients with chronic plantar fasciitis. Arch Phys Med Rehabil 98:2371-2377

12. Indikationen für Patienten: Digest e.V. [cited 2021 Feb 17]. Available from: https://digest-ev.de/patienten/indikationen-fuerpatienten.html

13. Cormie P, Zopf EM, Zhang X, Schmitz KH (2017) The impact of exercise on cancer mortality, recurrence, and treatment-related adverse effects. Epidemiol Rev 1-22. Available from: https:// academic.oup.com/epirev/article-lookup/doi/10.1093/epirev/ $\operatorname{mxx} 007$ 
14. Neo J, Fettes L, Gao W, Higginson IJ, Maddocks M (2017) Disability in activities of daily living among adults with cancer: a systematic review and meta-analysis. Cancer Treat Rev 61:94-106

15. Crevenna R, Kainberger F, Wiltschke C, Marosi C, Wolzt M, Cenik F, Keilani M (2020) Cancer rehabilitation: current trends and practices within an Austrian University Hospital Center ${ }^{*}$. Disabil Rehabil 42:2-7

16. Shapiro CL, Van Poznak C, Lacchetti C, Kirshner J, Eastell R, Gagel R et al (2019) Management of osteoporosis in survivors of adult cancers with nonmetastatic disease: ASCO clinical practice guideline. J Clin Oncol 37:2916-2946

17. Auersperg V, Trieb K (2020) Extracorporeal shock wave therapy: an update. EFORT Open Rev 5:584-592

18. Crevenna R, Mickel M, Keilani M (2019) Extracorporeal shock wave therapy in the supportive care and rehabilitation of cancer patients. Support Care Cancer 27:4039-4041
19. Gesslbauer C, Mickel M, Schuhfried O, Huber DF-X, Keilani M, Crevenna R. The effectiveness of focused extracorporeal shockwave therapy in the treatment of carpal tunnel syndrome. A randomized, placebo-controlled pilot study. Wien Klin Wochenschr. In press

20. Crevenna R, Mickel M, Schuhfried O, Gesslbauer C, Zdravkovic A, Keilani M. Focused extracorporeal shockwave therapy in physical medicine and rehabilitation. Curr Phys Med Rehabil Reports. In press

Publisher's note Springer Nature remains neutral with regard to jurisdictional claims in published maps and institutional affiliations. 